\title{
LA PORNOGRAPHIE SELON HILDA HILST
}

\section{Leonardo Alexander do Carmo Silva ${ }^{1}$}

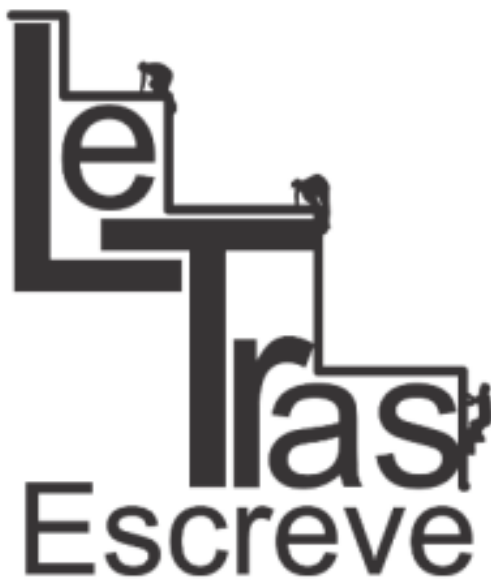

(ISSN 2238-8060)

Abstract: Contos d'Escárnio. Textos Grotescos is the second volume of the obscene tetralogy, a series of pornographic books published by Hilda Hilst between 1990 and 1992. The obscene project of the writer was a protest against the lack of visibility of her work. Although she was honored by critics, she had a very limited number of readers and she found it difficult to publish her books. Pornography, in her work, becomes a metaphor to the situation of the contemporary writer, who should "prostitute " himself to be able to live in this market. This paper studies how Hilda Hilst appropriates the main codes and clichés of pornography and transmutes them, creating a " misshapen pornography ».

Keywords: Hilda Hilst; Brazilian literature; Pornography; Humor

\section{Introduction - Qu'est-ce que la pornographie?}

Le roman Contos d'Escárnio. Textos Grotescos, de Hilda Hilst, est centré sur Crasso, le narrateur principal du roman et son auteur fictionnel. Le personnage est un sexagénaire qui décide de se lancer dans la littérature après avoir constaté la terrible qualité de ce qui

\footnotetext{
${ }^{1}$ Professor (attaché temporaire d'enseignement et de recherche) na Licence de Portugais da Université Sorbonne Nouvelle - Paris 3. Doutorando na Université Sorbonne Nouvelle - Paris 3 ; École Doctorale 122 - Europe Latine, Amérique Latine ; Centre de Recherches sur les Pays Lusophones (CREPAL), Paris, França - leonardoalexandersilva@gmail.com. Mestre em Literatura Comparada, pela Université Paris-Sorbonne, e mestre em Estudos Lusófonos, pela Université Sorbonne Nouvelle.
} 


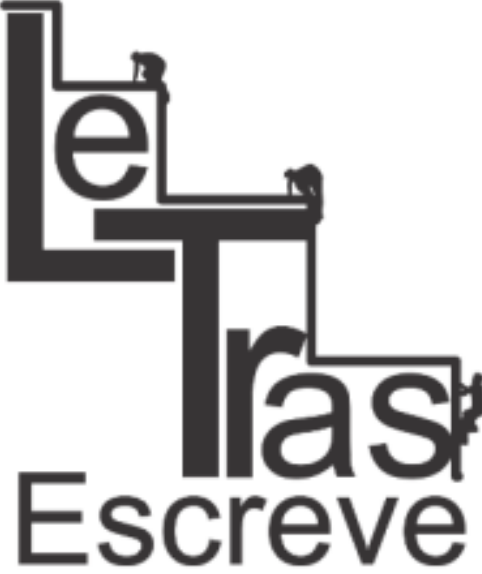

(ISSN 2238-8060)

était produit. Ironique, politiquement incorrect et grossier, il raconte ses principales aventures sexuelles et les efforts qu'il fait pour retrouver les textes perdus de Hans Haeckel, un écrivain existentialiste et sérieux qu'il a connu à travers Clódia, l'une de ses maîtresses. Le roman est l'assemblage du récit " autobiographique " de Crasso, enchâssé d'autres textes, comme les contes « fictionnels " du narrateur principal et les contes de Hans Haeckel.

Dans cet article, il s'agira de mettre en évidence la manière dont Hilda Hilst s'approprie les clichés et les codes de la pornographie conventionnelle et comment elle les adapte dans son texte. II sera important de se pencher tout d'abord sur la notion même de pornographie, sur ses fonctions et sur son statut.

Le premier usage attesté du mot " pornographe » en français remonte au XVIII ${ }^{\mathrm{e}}$ siècle avec la publication de Le Pornographe de Restif de la Bretonne, en 1769. Il ne s'agit pas d'un ouvrage licencieux, mais d'un traité à visée réglementariste et moraliste sur le contrôle de la prostitution par l'État à l'époque des Lumières. L'usage que fait l'écrivain français du mot est d'ailleurs très lié à son étymologie car, en grec ancien, « pornè » signifie prostituée. Selon Dominique Maingueneau, Restif de la Bretonne a dû s'inspirer du mot grec "pornographos», qui désignait l'auteur qui aborde le sujet de la prostitution, et du mot « pornographia » qui désignait un genre pictural caractérisé par la représentation des prostituées (MAINGUENEAU, 2007, p. 9).

Comme le souligne Lynn Hunt, dans son ouvrage The Invention of Pornography, bien que l'exhibition des organes génitaux et des actes sexuels soit très ancienne (pratique attestée à diverses époques et lieux de la planète), la pornographie ne s'est constituée comme une catégorie distincte dans le domaine de la littérature et de la re- 


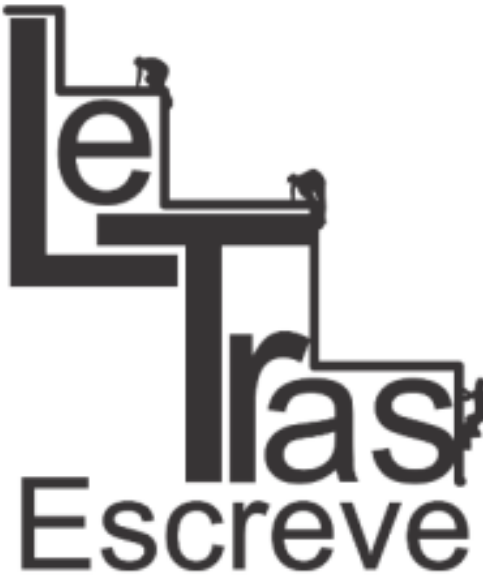

(ISSN 2238-8060)

présentation visuelle, qu'au $\mathrm{XIX}^{\mathrm{e}}$ siècle, moment où le mot " pornographie " commence à être utilisé dans le sens qu'il a aujourd'hui (HUNT, 1999, p. 10). La référence à la prostitution disparaît progressivement et le mot se met à désigner la représentation des « choses obscènes» (GOULEMOT, 1994, p. 19-28). L'écrivaine américaine ajoute que le développement de la pornographie moderne doit beaucoup à l'évolution de l'imprimerie et à la censure de l'Église qui a aidé à « institutionnaliser » la pornographie.

L'ouvrage Introduction à la pornographie, de Claude-Jean Bertrand et Annie Baron-Carvais donne la définition la plus consensuelle de la pornographie: "La pornographie représente, ou évoque clairement, un aspect de la nature, ou de l'activité sexuelle d'un ou plusieurs êtres humains. Et son effet principal (le seul parfois) est de stimuler la libido de l'usager, quelle que soit l'intention du créateur " (BERTRAND ; CARVAIS, 2001, p. 31). Dans cette définition le rôle du consommateur est mis en évidence. Cette notion de la pornographie est très proche de ce qu'affirme Hilda Hilst dans un entretien : "Uma criança pode ver uma situação sexual e não ter absolutamente nenhum choque com isso. Então é o seu olhar que vai discriminar o que é realmente pornô. Ele é que vai induzir você a ter uma situação excitante ou não » (HILST apud DINIZ, 2013, p. 144).

Le terme "érotique " (du grec érôs : " le désir amoureux ») a été largement employé pendant l'âge classique pour désigner, de manière un peu imprécise, les « choses d'amour " (GOULEMOT, 1994, p. 21). Les frontières exactes entre la pornographie et l'érotisme sont assez difficiles à définir, mais l'usage moderne qui est fait de ces deux mots montre que " chacune de ces notions se légitime en effet par le rejet de l'autre " (MAINGUENEAU, 2007, p. 26). L'érotisme est généralement valorisé par rapport à la pornographie, puisqu'il est associé

https://periodicos.unifap.br/index.php/letras

Macapá, v. 7, n. 3, 20 semestre, 2017 


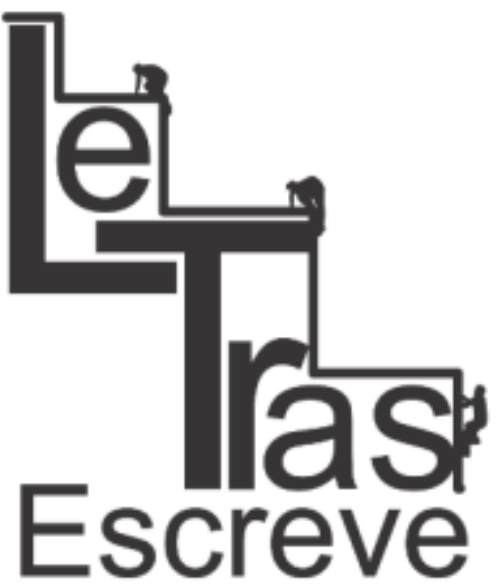

(ISSN 2238-8060)

à la recherche d'une beauté esthétique, à une ornementation du langage. Pour cette raison, il serait plus proche de la littérature. Outre par cet esthétisme, l'érotisme se distingue par une représentation de la sexualité humaine plus compatible avec les valeurs revendiquées socialement, constituant " une sorte de compromis entre la répression qu'impose le lien social et leur libre expression " (MAINGUENE$\mathrm{AU}, 2007$, p. 27). La pornographie, en revanche, est beaucoup plus directe; objective, dans son portrait des pratiques sexuelles; et agressive, visant ce que Maingueneau appelle l'« efficacité maximale » (MAINGUENEAU, 2007, p. 31).

Hilda Hilst est aussi connue pour ses poèmes érotiques. Dans son œuvre poétique, l'érotisme et le sacré sont souvent indissociables. Certains critiques voient d'ailleurs l'influence de Sainte Thérèse d'Avila et de ses chants mystico-érotiques dans l'œuvre de l'écrivaine brésilienne. II est intéressant de comparer un poème de Hilst, tiré du livre Do Desejo et un des courts poèmes enchâssés dans le récit de Contos d'Escárnio. Textos Grotescos:

Colada à tua boca a minha desordem./ O meu vasto querer./ O incompossível se fazendo ordem./ Colada à tua boca, mas descomedida/Árdua/ Construtor de ilusões examino-te sôfrega/ Como se fosses morrer colado à minha boca./ Como se fosse nascer/ $\mathrm{E}$ tu fosses o dia magnânimo/ Eu te sorvo extremada à luz do amanhecer. (HILST, 2004, p. 19)

Otávia tinha pelos de mel./ A primeira vez que me beijou a caceta/ Entendi que jamais seria anacoreta/ Não me beijou com a boca/ Me beijou com a boceta. (HILST, 2002, p. 15).

Cette comparaison nous permet de voir au sein de l'œuvre d'un même artiste deux pratiques très différentes : la première relève du registre érotique et travaille avec la suggestion ; le deuxième, du

https://periodicos.unifap.br/index.php/letras

Macapá, v. 7, n. 3, 2o semestre, 2017 


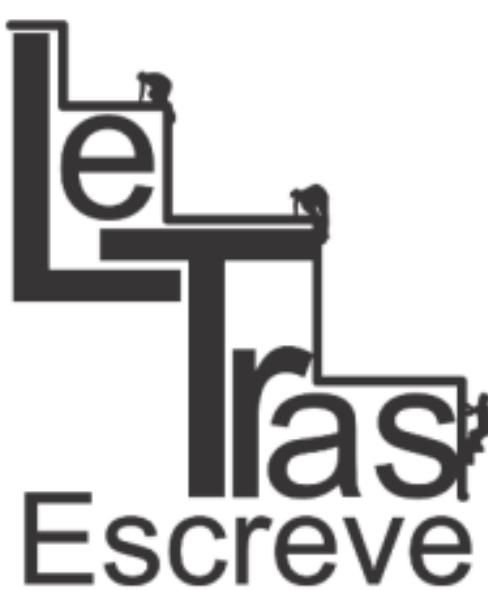

(ISSN 2238-8060)

registre pornographique, direct et moins sublimé. Ce qui prédomine dans Contos d'Escárnio. Textos Grotescos est la pornographie. Pour le projet de la tétralogie obscène, qui a une visée explicitement provocatrice, Hilst rejette l'atténuation propre à l'érotisme.

Le mot " obscène » est le plus utilisé pour décrire la phase de la carrière de Hilst qui correspond à la publication de la tétralogie, de 1990 à 1992. L'adjectif « pornographique » est, en revanche, employé avec prudence par la critique et par les commentateurs de l'œuvre de l'écrivaine. Même si « pornographie » est souvent associé à «obscène ", les deux mots ne sont pas équivalents. La pornographie, dans ses diverses manifestations, est perçue par certains comme étant forcement obscène. Néanmoins, toute obscénité n'est pas pornographique. Le mot « obscénité » implique un jugement moral : « L'obscénité choque la pudeur, le sens des convenances - mais d'ordinaire elle est de surcroît répugnante, car laide, sale ou vulgaire " (BERTRAND; CARVAIS, 2001, p. 30).

Étymologiquement, le mot « obscène » vient du latin « obscenus » qui signifie sinistre, dégoûtant, indécent. Ariel C. Arango suggère que le mot latin serait à l'origine une modification du vocable " scena » et qu'il désignerait donc ce qui est " hors de la scène ", ce qui ne peut pas être représenté sur scène, ce qui doit rester caché (ARANGO, 1991, p. 14). On pourrait argumenter que non seulement la tétralogie obscène, mais aussi une grande partie de la littérature de Hilda Hilst est, en effet, obscène, car fondamentalement « importune », donnant à voir ce qui est " indésirable », ce qui est censé rester dans l'ombre, la partie la moins acceptable et la moins tolérable de l'existence humaine. Toutefois, le terme "pornographique» se révèle plus pertinent pour qualifier spécifiquement les textes de la tétralogie, dont Contos d'Escárnio fait partie, notamment lorsqu'on

https://periodicos.unifap.br/index.php/letras

Macapá, v. 7, n. 3, 2o semestre, 2017 


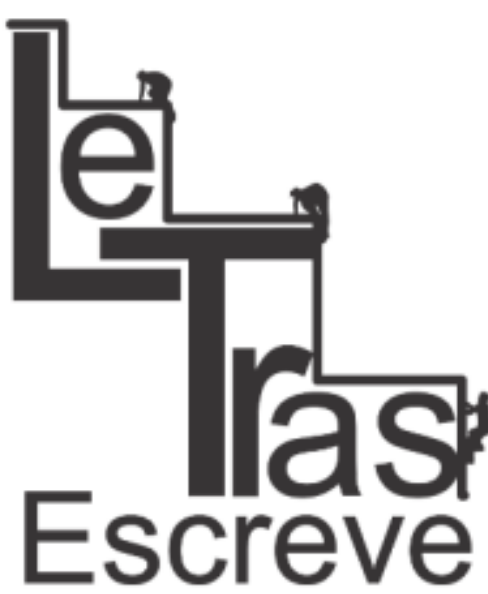

(ISSN 2238-8060)

s’intéresse au type de discours qu'il sollicite.

Il est important de souligner que ces étiquettes (« pornographique ", " érotique » et "obscène ») sont parfois attribuées de manière aléatoire et hypocrite. Ainsi, l'ouvrage d'un écrivain consacré par la critique sera difficilement classé comme pornographie, bien que son contenu soit effectivement pornographique : " D'ailleurs, dès qu'une œuvre montre du talent, personne n'ose plus l'appeler pornographique : par exemple, les romans de Françoise Rey, les BD de Manara ou les photos de Helmut Newton »(BERTRAND; CARVAIS, 2001, p. 157). On constate ce même phénomène dans l'appréciation qui est faite de l'œuvre de Hilst. La critique hésite au moment d'associer le projet de l'écrivaine à la pornographie. Plusieurs commentateurs de la tétralogie affirment que l'auteur n'a pas réussi à écrire ce type de texte à cause de la grande sophistication de son écriture. Il est important de rappeler que Hilst n'a pas hésité à utiliser le mot « pornographie » pour présenter les deux premiers romans de sa tétralogie dans un entretien donné à la TV Cultura, en 1990.

Alcir Pécora, I'un des grands spécialistes de l'œuvre de l'écrivaine, croit que le terme " pornographique " n'est pas approprié pour désigner l'œuvre de Hilst : « A sugestão de literatura pornográfica aplicada ao conjunto dos 4 textos, a rigor, também deve ser afastada como imprópria, a menos que os seus termos sejam redefinidos »(PÉCORA, 2010, p. 20). La position de Pécora est basée sur la définition usuelle de pornographie qui fait de l'excitation du lecteur l'effet ou le but principal du texte. Dans son célèbre essai The pornographic imagination, Sontag défend l'idée que ce critère n'est pas définitif pour distinguer un œuvre pornographique, d'une autre nonpornographique. L'essayiste rappelle que la sexualité humaine est très complexe et que le lecteur peut, par exemple, s'exciter à partir

https://periodicos.unifap.br/index.php/letras

Macapá, v. 7, n. 3, 2o semestre, 2017 


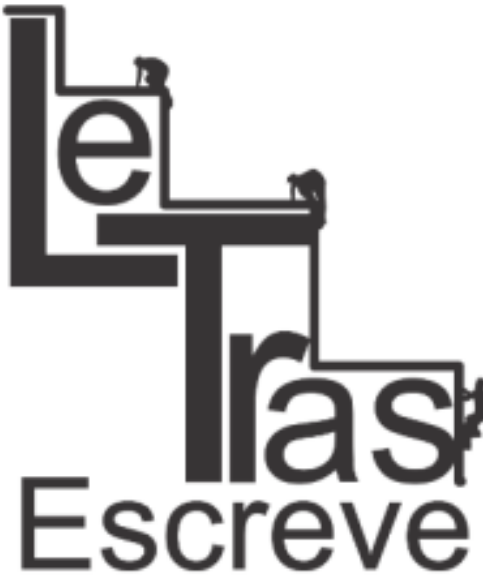

(ISSN 2238-8060) de la lecture d'un texte qui ne relève pas du tout de la pornographie (SONTAG, 1987). De plus, un texte pornographique n'est pas excitant pour tout lecteur.

La vocation transgressive de la pornographie, très présente dans le texte de Hilda Hilst, n'est pas un phénomène exclusif à la contemporanéité. La pornographie a été pendant longtemps un instrument de rébellion. Dans son livre The Invention of Pornography, Lynn Hunt affirme que, depuis le $X V I^{e}$ siècle, la pornographie est associée à la subversion politique et sociale. Les textes pornographiques et obscènes ont véhiculé pendant longtemps des critiques sociales, politiques et des idées philosophiques (par exemple, les plus célèbres, ceux de Sade). Ils étaient très souvent censurés et leurs auteurs persécutés. Cette fonction critique de la pornographie est devenue moins présente à partir du XIX ${ }^{\mathrm{e}}$ siècle, avec la pornographie moderne. Hilst dialogue, par conséquent, avec la tradition du " genre » pornographique, en utilisant la pornographie comme une façon de se positionner devant le monde, de montrer son insatisfaction, d'adresser des critiques aux éditeurs, à la société brésilienne et à ses propres lecteurs.

La pornographie a été historiquement et traditionnellement une affaire d'hommes. Pendant les années 70 et 80 , quelques mouvements féministes et ultra-féministes se sont lancés contre la pornographie, surtout aux États-Unis, argumentant que le discours pornographique véhiculait la haine masculine contre les femmes, souvent envisagées comme des objets sexuels soumis au désir phallique. Néanmoins, à partir de la même époque, les femmes ont commencé à produire elles-aussi de la pornographie. Hilda Hilst a été l'une des premières femmes écrivaines au Brésil à intégrer la pornographie dans le domaine de la « haute littérature ».

https://periodicos.unifap.br/index.php/letras

Macapá, v. 7, n. 3, 2o semestre, 2017 
La pornographie écrite brésilienne est encore un domaine peu étudié, ce qui peut révéler, d'une part, un manque d'intérêt pour ce « genre » marginalisé et d'une autre, le fait que la littérature pornographique au Brésil n'a pas connu un développement aussi important que dans d'autres pays, comme aux États-Unis et en France. Ce phénomène peut s'expliquer par la concurrence des ouvrages importés (la série française Emmanuelle, par exemple) et la concurrence d'autres médiums, comme le cinéma, avec la pornochanchada, le genre pornographique le plus typiquement brésilien, qui a eu beaucoup de succès dans les années 70 et 80 . La pornochanchada est la version érotisée de la chanchada, genre de spectacle théâtral ou filmique marqué par le registre burlesque, par l'humour et par la caricature. Il est possible de voir un rapport entre ce genre cinématographique et l'œuvre pornographique de Hilst, qui est aussi marquée par ces éléments.

La volonté de choquer et de scandaliser est associée dans le projet de Hilda Hilst à un désir de popularisation de son œuvre. Quand on lui a demandé si la pornographie était une stratégie de marketing, l'écrivaine a répondu : «É claro que sim, porque eu penso assim: É um absurdo você fazer obras-primas como eu faço e guardar tudo na gaveta, esperando que daqui a cinquenta anos as pessoas falem de você. O escritor, acima de tudo, quer ser lido » (HILST, 2013, p. 139). L'usage que fait Hilst de la pornographie nous fait penser à ce que dit Friedler à propos du récit pornographique, vu comme une forme essentielle de l'art pop, un type de divertissement plus proche du vice que de l'art. Ermelinda Ferreira ajoute, cependant, que le texte de Hilst oscille entre cette conception de la pornographie postmoderne et une attaque ironique, idéologique, qui semble parfois s'opposer au "genre » pornographique et à l'industrie culturelle dont il 


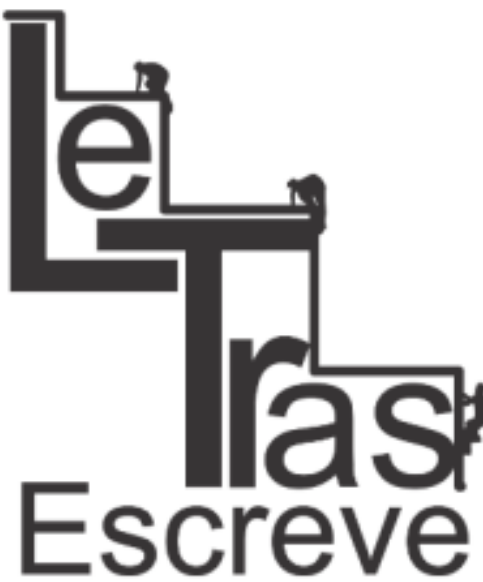

(ISSN 2238-8060)

fait partie (FERREIRA, 2003, p. 119).

Ainsi, le texte de Hilst se situe entre un hommage et une ridiculisation de la pornographie, ce qui nous renvoie à ce que dit Maingueneau à propos des manières dont les écrivains contemporains peuvent s'approprier le matériau pornographique. Pour lui, l'écrivain contemporain peut utiliser ce matériau d'une manière conventionnelle (" captation ») ou subvertir le dispositif pornographique (" subversion "), en le disqualifiant. II est possible de trouver dans Contos d'Escárnio. Textos Grotescos les deux opérations. On verra d'abord comment Hilda Hilst s'approprie les codes pornographiques et ensuite comment elle les déforme, en les mettant au service de l'univers qu'elle crée dans son roman.

\section{L'application des codes du discours pornographique}

Hilda Hilst considérait Contos d'Escárnio. Textos Grotescos plus "pornographique " que son roman précédent, O Caderno Rosa de Lori Lamby (HILST apud Diniz, 2013, p. 144). Dans le deuxième roman de la tétralogie obscène, Hilst s'approprie plus explicitement certains traits et clichés de la pornographie écrite. Si Lori Lamby péchait par son inexpérience (elle n'avait que huit ans), Crasso, l'auteur fictionnel de Contos d'Escárnio, correspond davantage au profil du pornographe. Le roman de Hilda Hilst se sert d'ailleurs d'un dispositif typique des récits pornographiques : l'adoption d'un narrateur homodiégétique qui raconte ses expériences sexuelles. D'autres caractéristiques rendent ce narrateur encore plus conforme à l'univers pornographique conventionnel : il appartient au sexe masculin, il est hétérosexuel et il véhicule un regard machiste et phallocentrique : " $O$ que eu podia fazer com as mulheres além de foder? » (HILST, 2002, p. 18).

https://periodicos.unifap.br/index.php/letras

Macapá, v. 7, n. 3, 20 semestre, 2017 
Selon Goulemot, le narrateur d'un texte pornographique a un "double rôle » : il décrit ce qu'il voit et il se décrit en train d'agir. II doit jongler avec une vision interne, dominée par ses affects et sensations, et une vision externe qui permet au lecteur de se représenter un « tableau » (GOULEMOT, 1994, p. 159). À ce propos, Maingueneau affirme que les séquences pornographiques peuvent être construites de manière à ce que la dimension picturale prédomine, à ce que les affects prédominent, ou de manière à ce qu'il y ait un équilibre entre les deux (MAINGUENEAU, 2007, p. 63). Dans Contos d'Escárnio, c'est la volonté de dresser un "tableau » qui l'emporte. La description de l'acte sexuel est mise en évidence, au détriment des affects des personnages: "Como aquela punheta a quatro mãos não dava certo, espremi minha cara entre os dois suculentos melões e fui metendo desengonçado e suarento. Ela não dava um pio. Nem suspirava nem gemia » (HILST, 2002, p. 15-16).

Dans l'épisode consacré à Liló, Hilda Hilst construit un tableau à plusieurs niveaux, du micro (le gros plan sur l'organe sexuel féminin, pour utiliser le langage cinématographique) au macro (au plan général avec l'ajout des nouveaux acteurs dans la scène). Le lecteur peut (ISSN 2238-8060) ainsi accéder à la "dimension configurationnelle de la scène ", c'està-dire, il peut visualiser mentalement la façon dont les personnages occupent cet espace. La " mise en tableau » est essentielle dans les textes pornographiques pour que le lecteur puisse se représenter de manière précise l'acte sexuel. La description est, par conséquent, le dispositif le plus sollicité dans ces textes. Dans la scène du bordel, chaque geste de Liló mérite une description détaillée : «la empurrando o tecido da calcinha para a virilha da mulher e esticava os pentelhos devagar » (HILST, 2002, p. 28).

Dans cet épisode, l'essence même du dispositif pornographi-

https://periodicos.unifap.br/index.php/letras

Macapá, v. 7, n. 3, 2o semestre, 2017 


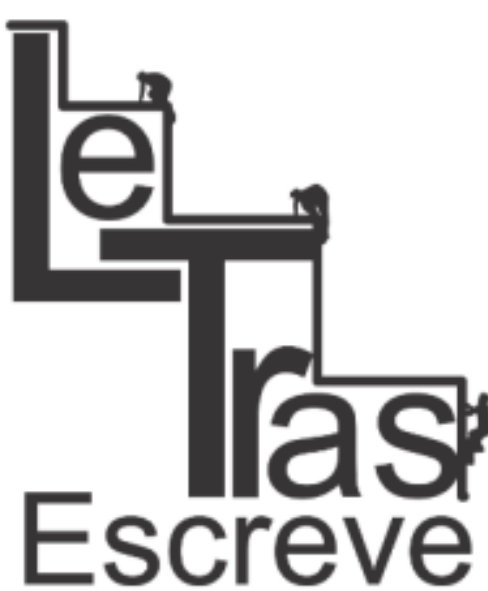

(ISSN 2238-8060)

que, qui consiste à montrer ce qui appartient au domaine privé, est métaphorisé par l'exhibitionnisme de Liló et par le voyeurisme des autres personnages: " No bordel todo mundo gostava de ver Liló lamber as putas. E ele adorava que o vissem » (HILST, 2002, p. 27). Dans les récits pornographiques, le narrateur occupe souvent la position de voyeur. Crasso, le narrateur principal du roman, est un des témoins de cette scène. Le narrateur décrit non seulement la performance de Liló, mais aussi la réaction du « public ». Au contraire de ce qui arrive dans les scènes où il est l'un des acteurs, le regard du narrateur ne peut être qu'extérieur, il se retrouve ainsi dans une position analogue à celle du lecteur, la position du voyeur. Selon Goulemot, le lecteur des textes pornographiques est inévitablement un voyeur, parce que ce type de texte est fondé sur le regard et il donne à voir par l'écriture. Le regard du voyeur est stimulant parce qu'il transgresse les limites, envahit la zone du privé et de l'interdit.

L'épisode de Liló est celui qui se rapproche le plus des récits pornographiques conventionnels. II maintient sa charge sexuelle constante et la progression du texte accompagne la montée du désir des personnages jusqu'à la jouissance. Cet épisode joue également avec l'un des clichés des textes pornographiques, la fausse résistance de la femme : «O prazer de Liló era o acanhamento postiço da muIher. Todas sabiam que ele só gostava se a mulher fingisse pudor, um pouco de receio no início, um tantinho de apreensão » (HILST, 2002, p. 28). Goulemot explique que le récit pornographique ne met jamais en scène une vraie résistance à l'acte d'aimer : « S'il évoque une résistance, c'est pour mieux peindre une ébauche de viol, espèce de piment, par ailleurs sans grande conséquence, puisque la victime devient vite consentante et finit par partager la jubilation et les plaisirs de son agresseur » (GOULEMOT, 1994, p. 67).

https://periodicos.unifap.br/index.php/letras

Macapá, v. 7, n. 3, 2o semestre, 2017 
La scène du bordel nous renvoie à une des principales caractéristiques de la pornographie : son caractère spectaculaire. Selon Maingueneau, " l'activité sexuelle qui est montrée doit d'une manière ou d'une autre être spectaculaire, et ceci en deux sens : a) elle doit constituer un spectacle, b) elle doit échapper à l'ordinaire, être sexuellement performante » (MAINGUENEAU, 2007, p. 34). Cette dimension spectaculaire est présente dans plusieurs séquences pornographiques du roman. Les rapports sexuels de Crasso avec Flora, Otávia, Josete et les rêves érotiques du narrateur n'ont rien d'ordinaire. Un des procédés utilisés par l'auteur pour accentuer le caractère spectaculaire des scènes est l'usage de l'hyperbole et de l'exagération : « Mas nenhuma outra mulher era dona desse gorgolejo na garganta. Era mais do que uma rosnada langorosa. Vinha do fundo de águas negras, mas era também pungente e prazeroso. Como se você estivesse fodendo uma onça-mulher filhote » (HILST, 2002, p. 17).

Les personnages du roman qui appartiennent à l'univers pornographique sont très performants en matière de sexe : "ils ne sont que corps offerts, désirs spontanés et voluptés immédiates » (GOULEMOT, 1994, p. 67). Les personnages féminins, en particulier, font preuve d'une libido extraordinaire. C'est le cas de Josete qui peut avoir des orgasmes successifs: « Depois de Josete ter gozado umas dez vezes entre sabiás e musses e álcoois dos mais finos que me custavam um caralhão de dinheiro, levantava-se garbosa, Espártaco antes da derrocada final, naturalmente » (HILST, 2002, p. 20).

Comme la plupart des textes pornographiques, Contos d’Escárnio. Textos Grotescos obéit au principe de la « sexualité phallique » : " "Le "récit" pornographique se structure selon les contraintes d'une sexualité phallique qui investit méthodiquement l'intervalle qui va de la naissance du désir à sa satisfaction. " (MAINGUENEAU, 
2007, p. 45). Écrit traditionnellement par et pour les hommes, ces textes, comme l'expliquent Bertrand et Carvais, mettent en scène des personnages féminins " dont la sexualité ressemble à celle des mâles : disponibles à tout moment et pour tout mâle, extatique à la moindre caresse, prêtes à avoir n'importe quel rapport sexuel " (BERTRAND ; CARVAIS, 2001, p. 167). Les femmes représentées dans le roman de Hilst suivent ce modèle : elles ont un appétit sexuel insatiable. Une scène d'un conte de Crasso illustre bien ce phénomène ; le personnage féminin, $\mathrm{Ma}$, change tranquillement de partenaire pendant l'acte sexuel lorsqu'un inconnu surgit dans la scène : « Ma abriu seus adoráveis olhos de um verde de folhinhas novas, abriu também suas deleitáveis coxas e disse rouquenha: vem também, grandão, vem. Fiquei perplexo » (HILST, 2002, p. 107).

Le principe de la sexualité phallique apparaît également dans le grand intérêt que la pornographie porte à la satisfaction du désir, au détriment de tout ce qui entoure la naissance de ce désir : les affects, les préliminaires, le processus de séduction, tout ce qui renvoie à une dimension plus féminine de la sexualité. Dans Contos d'Escárnio, ce phénomène se manifeste par une sorte d'obsession de l'orgasme. Dans le roman, les personnages jouissent tout le temps et leurs orgasmes sont puissants: "Assim que esporrei quis ver a cara de Lina " (HILST, 2002, p. 16) ; " esporrei pela segunda vez, rápida e fartamente " (HILST, 2002, p. 16) ; "Bina gozava na boca de Liló » (HILST, 2002, p. 29) ; « Aí ela começou a gozar » (HILST, 2002, p. 76) ; «Gozei grosso e quente » (HILST, 2002, p. 88). Dans un conte de Crasso, le culte de l'orgasme est poussé jusqu'à ses limites. Le personnage masculin demande à sa partenaire combien elle veut pour se laisser percer à coups de couteau pendant l'acte sexuel. Elle répond : " se eu gozar quero nada não, bem » (HILST, 2002, p. 91). 


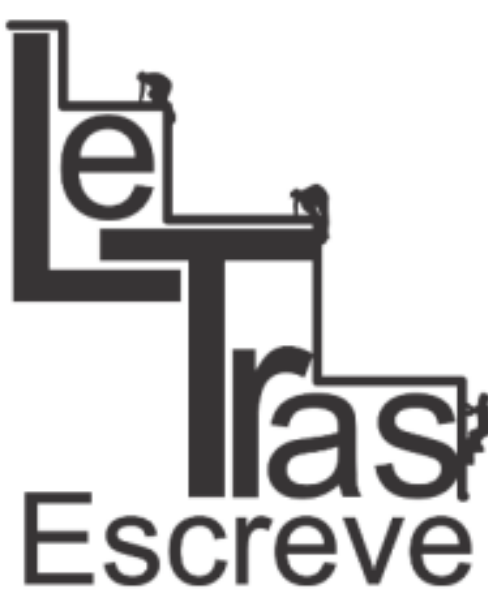

(ISSN 2238-8060)
La plupart des personnages du roman sont soumis à leurs propres désirs. Ils ne sont pas dotés d'une psychologie approfondie et ils se différencient par leurs excentricités. Maingueneau observe que les personnages des récits pornographiques disposent rarement d'un nom complet. Ils peuvent être désignés par leur prénom, par un surnom, par un simple pronom (comme « il » et " elle »), par des initiales, ou par un nom fantaisiste. Dans Contos d'Escárnio, tous les personnages sont désignés par leur prénom (Crasso, Clódia, Otávia, Flora, Josete, Lina, Líria, etc), sauf une exception très significative : Hans Haeckel. II est cohérent que ce personnage, l'écrivain sérieux et existentialiste, soit l'exception car, au contraire des autres, il n'appartient pas à l'univers pornographique. Maingueneau rappelle que « le patronyme dit une origine, une famille, des ancêtres, une insertion sociale; le prénom, en revanche, est lié à l'intimité, il individualise sans individualiser, puisque dans une collectivité toutes sortes de gens ont le même prénom " (MAINGUENEAU, 2007, p. 56). On a donc des personnages qui ne sont pas dotés d'une vie sociale, ils font partie d'un univers particulier où le sexe est la raison et le but de tout.

Autre caractéristique des récits pornographiques incorporée dans le texte de Hilst est la résistance à la notion de couple et à tout sentimentalisme : « Eu era o fixo. Mas a alminha de Clódia era brejeira, velhaca e sensual. Quando fizemos o trato do amor livre ela explicou: a rotina, a mesma paisagem das genitálias, faz apodrecer a sensualidade» (HILST, 2002, p. 42). L'interchangeabilité des couples est I'un des impératifs du récit pornographique. L'amour n'est d'ailleurs pas perçu comme un pré-requis pour l'activité sexuelle, au contraire, il peut inhiber l'excitation: "Será que ando sentindo amor? Meu Deus, isso vai me brochar para sempre » (HILST, 2002, p. 87). L'amour et le sexe sont souvent incompatibles ou dissociables dans l'univers

https://periodicos.unifap.br/index.php/letras

Macapá, v. 7, n. 3, 2o semestre, 2017 


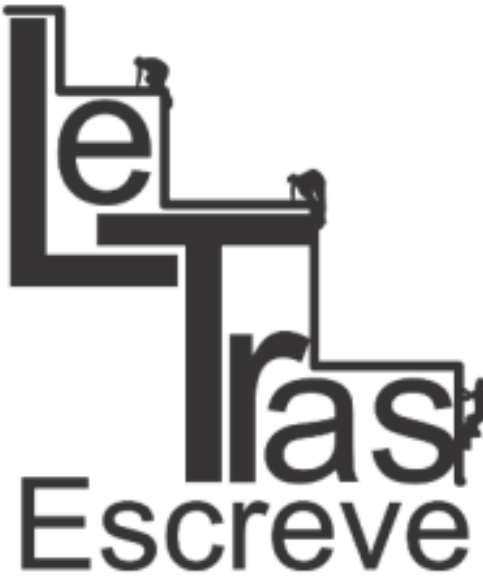

(ISSN 2238-8060)

pornographique. Le début du roman montre parfaitement qu'il n'y a pas de place pour le sentimentalisme dans le récit. Même lorsque le narrateur parle d'un traumatisme, l'élément pornographique intervient : «Todo mundo quando me via dizia: lá vai o Crasso, filho daquela da crassa putaria. Eu ficava com os olhos úmidos mas logo em seguida, apesar da minha timidez, mostrava o pau » (HILST, 2002, p. 14).

L'insertion inusitée du mot "pau » dans cet extrait nous fait réfléchir à l'usage du langage obscène dans le roman. Selon Maingueneau, le roman pornographique se caractérise par l'usage de mots qui sont largement sous l'emprise de tabous. Ce type de texte fait passer pour normal un type de langage qui est presque interdit dans la vie quotidienne. Le pouvoir de stimulation sexuelle de ces mots " maudits" se trouve justement dans leur caractère transgressif. Dans son roman, Hilda Hilst emploie une quantité considérable de mots obscènes, comme "foder ", "pau », "boceta ", "cu », " pica ", parmi d'autres.

Jusqu'à l'apparition de Hans Haeckel dans le roman, le récit adopte une structure épisodique, commune aux textes pornographiques. II s'agit d'une juxtaposition d'épisodes indépendants, ou presque indépendants, qui constituent un inventaire de pratiques sexuelles. Ce type de structure permet à Crasso de raconter ses aventures lubriques les plus extraordinaires. Dans chacune de ces histoires, il a un rapport avec une femme différente : Lina, la vierge ; Otávia, la masochiste ; Flora, la cultivée ; et Josete, l'excentrique. Outre ces aventures personnelles, le narrateur raconte aussi l'épisode de Liló et celui de son oncle Vlad (un pédophile).

Hilda Hilst s'inspire de conventions et de codes de la pornographie dans son roman. Néanmoins, elle procède également à une déformation du discours pornographique conventionnel. Nous ver-

https://periodicos.unifap.br/index.php/letras

Macapá, v. 7, n. 3, 20 semestre, 2017 
rons dans la suite comment l'auteur crée ce que Goulemot appelle un « texte pornographique impur » (GOULEMOT, 1994, p. 95).

\section{La « déformation " de la pornographie}

Dans son ouvrage, Ces livres qu'on ne lit que d'une main, JeanMarie Goulemot montre quels peuvent être les " échecs » d'un texte pornographique, c'est-à-dire, quels procédés éloignent ce type de texte de son but principal, l'excitation physique du lecteur. Parmi ces défauts, on trouve: I'utilisation excessive de la métaphore et de l'ironie; la présence de pauses et de digressions et l'évocation de comportements sexuels pervers ou trop excentriques. Le texte d' Hil-

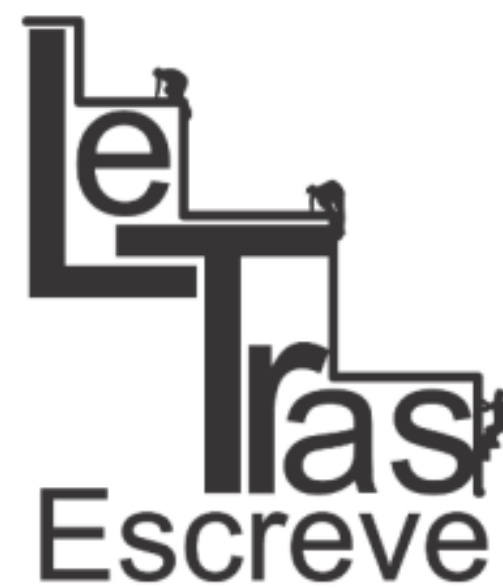

(ISSN 2238-8060) da Hilst se caractérise par tous ces procédés.

L'aspect digressif du discours de Crasso est l'un des éléments qui déforment le discours pornographique. L'auteur fictionnel du roman interrompt souvent la scène ou l'épisode qu'il raconte pour introduire un commentaire qui n’a rien d'érotique : " A primeira vez que "a fodi" (ou que "fodia" ou que "fui fodê-la", é melhor?) enganeime na tradução de seu breve texto "(HILST, 2002, p. 16-17). Le commentaire métatextuel attire l'attention du lecteur sur l'élaboration du texte et dévie son attention de la scène. Dans l'épisode de Liló, celui qui se rapproche le plus de la pornographie conventionnelle, il y a aussi une brève digression : « As mulheres seguravam a cabeça da que estava sendo chupada e alguns homens a beijavam na boca outros nos seios. Tinha jeito de mesa de cirurgia aquilo tudo (sorry, médicos) » (HILST, 2002, p. 29). Le narrateur interrompt la description pour ajouter un commentaire ironique, à priori inutile, qui crée une petite pause humoristique. Or, le rire n'est généralement pas l'effet souhaité par la pornographie car il signale que le 


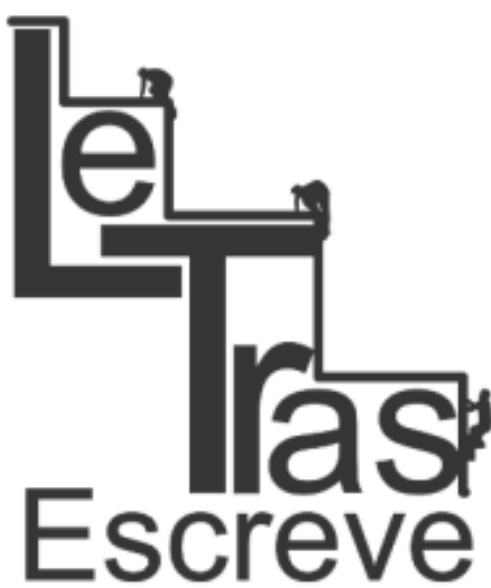

(ISSN 2238-8060)

lecteur n'a pas adhéré à la scène du point de vue érotique.

Tout au long du roman, le narrateur multiplie les commentaires « inopportuns », humoristiques, qui contrastent avec la dimension pornographique du texte : "Sorrindo soltava um pífio arroto de tordos e ostras abafado entre os seus dois dedinhos, enquanto os meus (dedos, naturalmente) beliscavam-Ihe a cona. [...] ela gozava escondendo o gozo e simulando um segredo e enchendo de bafo, gemidos e saliva a concha do meu ouvido » (p. 20). La description pornographique est " ruinée » par la présence d'éléments étrangers à ce type de discours : la parenthèse explicative et les mots " arroto " et « bafo ", qui renvoient à des phénomènes non-érotiques, voire même répulsifs.

Le passage où Crasso raconte à Clódia comment il s'est stimulé sexuellement est révélateur du type de rapport qu'il entretient avec le sexe : «Depois acrescentei a Santa Teresa do Bernini, aqueles pés em ponta recebendo as flechadas da beleza e gozando gozando. Te vesti de carmelita, querida. Me vesti de Sátiro. Cornos e tudo » (HILST, 2002, p. 88). Il s'agit d'un rapport non-conventionnel qui passe par l'association d'éléments à priori incompatibles ou hétérogènes. L'inclusion d'une sainte catholique et d'un personnage mythologique dans le rêve érotique montre à quel point Crasso brouille les limites entre l'univers du sexe, du savoir et de la religion.

Dans Contos d'Escárnio, Hilda Hilst joue délibérément avec les limites de la pornographie conventionnelle. Dominique Maingueneau distingue trois types de pornographie: la pornographie canonique, la plus consommée et acceptée socialement; la pornographie tolérée, qui montre des pratiques perçues comme anormales; et la pornographie interdite, qui véhicule des pratiques criminelles (comme le viol et la pédophilie) (MAINGUENEAU, 2007, p. 42). La dimension provo-

https://periodicos.unifap.br/index.php/letras

Macapá, v. 7, n. 3, 2o semestre, 2017 


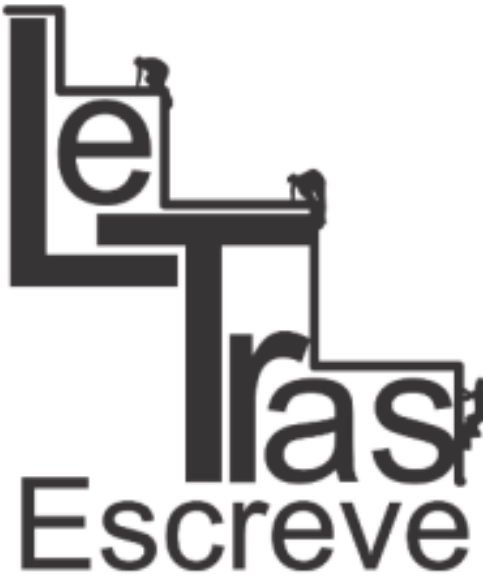

(ISSN 2238-8060) catrice du texte de Hilst doit beaucoup au choix de l'auteur de jouer avec les anormalités et les interdits, comme la pédophilie, déjà présente dans son roman précédent. Dans Contos d'Escárnio, l'oncle du narrateur principal a une relation avec un jeune adolescent: "Tio Vlad segurava a escada para Tavim e vi quando o moço ia descendo e a cara de tio Vlad afundando nas nádegas do dito cujo, o nariz enterrado no rego da calça da beleza » (HILST, 2002, p. 24).

La pédophilie dans le récit sert à choquer le lecteur et à affronter les mœurs du public. Ce goût pour le " politiquement incorrect » apparaît également dans la conversation du narrateur avec le diable à la fin du roman : "Diz que gostaria de ser humano para poder publicar um livro e colocar o retratinho dele, criança, na contracapa. DigoIhe que as criancinhas de hoje gostam mesmo é de enfiar o dedo no cu. Ele fica alarmado. É mesmo? pergunta » (HILST, 2002, p. 110). Crasso, ce grand immoral, réussi même à choquer le Diable.

Outre la pédophilie, Hilda Hilst s'intéresse à d'autres interdits et tabous comme la zoophilie et l'inceste. La zoophilie apparaît dans le conte de Hans Haeckel, intitulé "Lisa ", où un homme entretient une relation amoureuse et sexuelle avec un singe, le personnage éponyme du texte: "Então vi: o homem nu, deitado, e Lisa acariciando-lhe o sexo com as mãozinhas escuras, delicadas " (HILST, 2002, p. 45). Dans Caderno Negro, récit enchâssé de $O$ Caderno Rosa de Lori Lamby, il y a aussi une scène de zoophilie, plus perverse et violente. Dans "Lisa ", la tendresse entre le personnage (incapable de créer des liens avec les autres humains) et l'animal cause l'épouvante du narrateur du conte, celui qui regarde la scène.

L'inceste est exploré dans le dernier conte de Crasso. L'initiation sexuelle est l'un des grands topos de la littérature pornographique. Dans ce conte, Hilst subvertit ce lieu commun : c'est la

https://periodicos.unifap.br/index.php/letras

Macapá, v. 7, n. 3, 20 semestre, 2017 


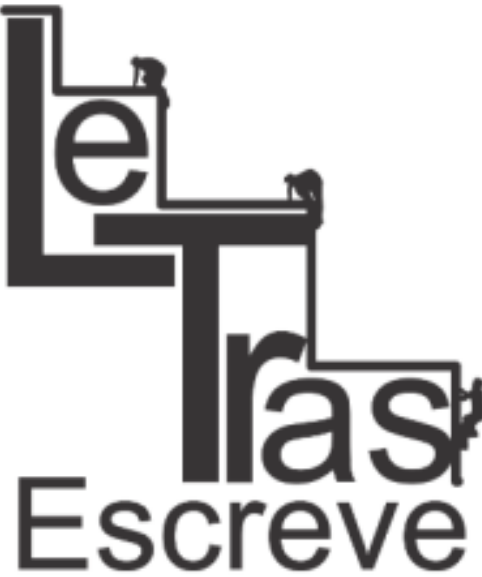

(ISSN 2238-8060)

mère qui entraîne son fils dans l'univers du sexe. «Quando completei 16 anos, ela, sabedora do meu infortúnio, sentou-se na sua linda poltrona de cetim perolado, abriu as magníficas coxas rosadas e colocando um cacho de uvas purpúreas nos seus meios sagrados, disseme: chupe-as, até encontrar o paraíso » (HILST, 2002, p. 105). Le récit pornographique de Hilda Hilst transgresse les limites de ce qui est socialement acceptable. Comme le souligne Eliane Robert Moraes, Hilst crée une pornographie incontrôlée, qui excède les normes du marché (MORAES, 2007, p. 13).

Outre les interdits, le roman pornographique de Hilst évoque des comportements bizarres ou non-conventionnels. Flora rit au moment de jouir et elle aime déclamer Lucrèce pendant l'acte sexuel : « [Flora] citava Lucrécio enquanto me afagava os culhões e encostava nas bochechas translúcidas a minha caceta» (HILST, 2002, p. 18). Otávia aime la violence physique: "Meti-Ihe a mão na cara quatro, cinco vezes. Otávia rosnava langorosa. A cada bofetão um ruído grosso » (HILST, 2002, p. 17). Clódia aime insérer des légumes dans son vagin et sucer les doigts d'hommes noirs: « Aquece alguns rabanetes, aqueles compridinhos para eu te pôr no buraco, como gostas, as casquinhas vermelhas ao redor como flor, como gostas " (HILST, 2002, p. 82).

Comme dans la pornographie conventionnelle, le roman de Hilst emploie des mots et des expressions considérés comme obscènes et vulgaires (" pau " et " boceta ", par exemple, sont largement sollicités). Néanmoins, l'accumulation et la variété de termes utilisés pour désigner les organes génitaux, quelques uns étant inusités ou créatifs, finit par accentuer la dimension comique du texte. Dans le roman, on trouve un véritable inventaire de termes obscènes : « pomba », « xereca », « cona », « perseguida », « crica », « vara », «

https://periodicos.unifap.br/index.php/letras

Macapá, v. 7, n. 3, 20 semestre, 2017 
caceta ", " caralho ", " talo », " mastruço », " pica », pour citer quelques exemples.

Ces mots et expressions vulgaires coexistent avec un registre parfois très soutenu, littéraire ou même scientifique, ce qui crée un effet de contraste et d'hétérogénéité. Cette hybridité peut être vue, par exemple, dans un court poème de Crasso :

Posso dobrar joelhos e catar pentelhos?/ Posso ver o caralho do emir/ E a "boceta de mula" (atenção: é uma planta da família das esterculiáceas)/ Que acaba de nascer no jardim do grão-vizir?/ Devo comprimir junto ao meu palato o teu régio talo?/ Ou oscular tua genitália/ dulçorosa Vestália? (HILST, 2002, p. 36)

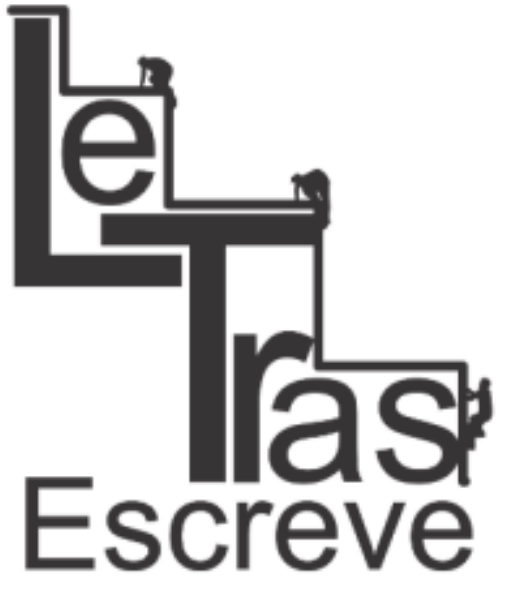

(ISSN 2238-8060)

Dans ce poème, les mots vulgaires (comme "caralho » et "penteIhos ") sont mis en relation avec des mots très soutenus (" oscular ", "régio ", "dulçorosa »), mots qui viennent du domaine de l'anatomie (« palato »), de la botanique (« talo ») et des noms scientifiques (« esterculiáceas »).

Un autre "défaut " du texte pornographique de Hilst est I'usage excessif du langage poétique : «Par nature, la poésie met en effet au premier plan la matérialité du signifiant verbal, là où l'écriture pornographique privilégie la transitivité du langage, censé s'effacer devant les spectacles qu'il donne à voir " (MAINGUENEAU, 2007, p. 15). Le langage poétique est sollicité dans tout le roman, même dans les parties en prose, comme on peut le vérifier dans l'usage que l'auteur fait des métaphores: "Enfio minha cabeçaabóbora candente entre as venosas virilhas de Clódia » (HILST, 2002, p. 46); « Blümschen, sonho com as tuas coxas carnudas e minha cabeça metida na tua vasta orquídea » (HILST, 2002, p. 82) ; «A leoa faminta caminha vagarosa, dourada, a úmida língua nas beiçolas cla- 
ras! » (HILST, 2002, p. 36). Hilda Hilst, poète avant tout, défie la transitivité du langage pornographique et attire l'attention du lecteur sur « la matérialité du signifiant verbal » en jouant avec le rythme, les sonorités des mots et les figures de style.

\section{Conclusion}

La pornographie chez Hilda Hilst se caractérise par l'association d'éléments stimulants (le dénudement des corps, la représentation de l'acte sexuel, le langage obscène) avec d'autres, au contraire, nonstimulants (les comportements répulsifs, inappropriés ou moralement inacceptables); par le mélange de registres; par les pauses dans le récit ; par l'usage de l'humour et du langage poétique. La pornographie devient chez Hilst le prétexte à une réflexion sur la production et la réception littéraires. Le choix du discours pornographique doit beaucoup à son pouvoir transgressif, de scandale. L'écrivaine crée d'ailleurs une pornographie démesurée qui va au-delà des pornographies conventionnelles en jouant avec les interdits (la pédophilie, l'inceste, la zoophilie), les absurdités et les bizarreries. À l'époque de la publication de la tétralogie, le scandale a été aggravé par le fait que Hilda Hislt était une femme (la pornographie étant historiquement une affaire d'hommes) sexagénaire et une poète consacrée par la critique, une écrivaine qui avait dédié plusieurs décennies de sa carrière à des thèmes " sérieux ", existentialistes.

\section{Références}

ARANGO, Ariel C. Os Palavrões. São Paulo: Brasiliense, 1991.

BERTRAND, Claude-Jean; BARON-CARVAIS Annie. Introduction à la 
pornographie, Panorama critique. Paris : La Musardine, 2001.

DINIZ, Cristiano (org.). Fico besta quando me entendem: entrevistas com Hilda Hilst. São Paulo: Biblioteca Azul, 2013.

FERREIRA, Ermelinda. Da poesia erudita à narrativa pornográfica: sobre a incursão de Hilda Hilst no pós-modernismo. Estudos de Literatura Brasileira Contemporânea. no 21. Brasília : janvier/juin 2003, p. 113-127.

GOULEMOT, Jean M. Ces livres qu'on ne lit que d'une main, Lecture et lecteurs de livres pornographiques au XVIII ${ }^{e}$ siècle. Paris : Minerve, 1994.

HILST, Hilda. Contos d'Escárnio, Textos Grotescos [São Paulo, Siciliano, 1990]. São Paulo: Globo, 2002.

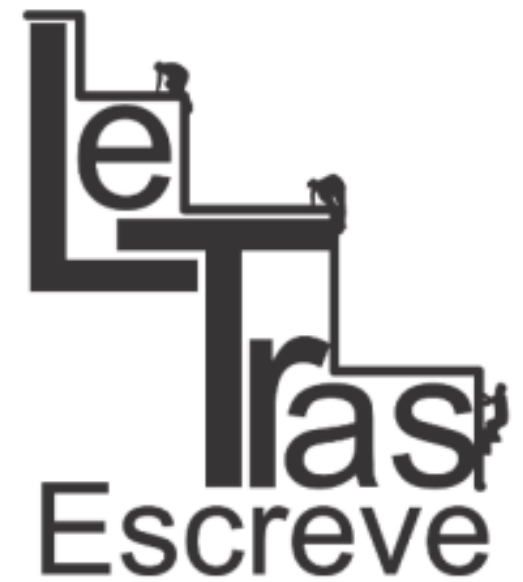

(ISSN 2238-8060)

HILST, Hilda. Do Desejo [Campinas, Pontes, 1992]. São Paulo: Globo, 2001.

HUNT, Lynn (org.), A Invenção da Pornografia : obscenidades e as origens da modernidade, 1500-1800 [1993]. Trad. Carlos Szlak. São Paulo : Hedra, 1999.

MAINGUENEAU, Dominique. La Littérature pornographique. Paris : Armand Colin, 2007.

MORAES, Eliane Robert. A Prosa Degenerada de Hilda Hilst. In: PRZYBYCIEN, Regina; GOMES, Cleusa (Orgs.). Poetas mulheres que pensaram o século XX. Curitiba: Editora UFPR, 2007, p. 11-15.

PÉCORA, Alcir (org.). Por que ler Hilda Hilst. São Paulo: Globo, 2010.

SONTAG, Susan. A imaginação pornográfica. In: A Vontade Radical [1969], Rio de Janeiro: Cia. das Letras, 1987.

Artigo Recebido em 15/02/2017

Aceito em 16/06/2017 